\title{
Antitumor activity of curcumin by modulation of apoptosis and autophagy in human lung cancer A549 cells through inhibiting PI3K/Akt/mTOR pathway
}

\author{
FURONG LIU ${ }^{1}$, SONG GAO ${ }^{1}$, YUXUAN YANG ${ }^{1}$, XIAODAN ZHAO $^{1}$, YAMENG FAN $^{1}$, \\ WENXIA MA $^{1}$, DANRONG YANG ${ }^{1}$, AIMIN YANG ${ }^{2}$ and YAN YU ${ }^{1}$ \\ ${ }^{1}$ School of Public Health, Xi'an Jiaotong University Health Science Center; ${ }^{2}$ Department of Nuclear Medicine, \\ The First Affiliated Hospital of Xi'an Jiaotong University, Xi'an, Shaanxi 710061, P.R. China
}

Received August 4, 2017; Accepted December 29, 2017

DOI: 10.3892/or.2018.6188

\begin{abstract}
Curcumin is known to exhibit anticancer effects on various cancers with selective cytotoxicity in tumor cells. In the present study, the effects of curcumin-induced multiple PCDs on human non-small cell lung cancer (NSCLC) cells and the potential molecular mechanisms of apoptosis and autophagy triggered by curcumin via the PI3K/Akt/mTOR signaling pathway were explored, further confirmed by co-culture of curcumin with mTOR blocker rapamycin and PI3K/Akt inhibitor LY294002. The anti-proliferation effect of different stimulus was measured by MTT assay. Apoptosis was detected by flow cytometry. Autophagy induction was detected by MDC labeling and western blotting of Beclin1, LC3, and p62 expression. The mRNA and protein expression levels of Akt and mTOR were assayed by real-time fluorescence quantitative (qRT-PCR) technique and western blotting. Our results showed that curcumin inhibited the viability of A549 cells time- and dose-dependently. In addition, a dosage-dependent A549 cell apoptosis-induction phenomena was observed by the curcumin intervention. Moreover, obvious autophagy was induced after curcumin-treatment, characterized by the formation of fluorescent particles [autophagic vesicles (AVs)] and significant increase in ratio of LC3-II/LC3-I and Beclin1 as well as decreased p62 expression. Furthermore, the effect of curcumin on a substantial downregulation of phosphatidylinositol 3-kinase (PI3K)/Akt/mammalian target of
\end{abstract}

Correspondence to: Professor Yan Yu, School of Public Health, Xi'an Jiaotong University Health Science Center, 76 West Yanta Road, Xi'an, Shaanxi 710061, P.R. China

E-mail: yuyan@mail.xjtu.edu.cn

Professor Aimin Yang, Department of Nuclear Medicine, The First Affiliated Hospital of Xi'an Jiaotong University, 277 West Yanta Road, Xi'an, Shaanxi 710061, P.R. China

E-mail: yangaimin@mail.xjtu.edu.cn

Key words: curcumin, apoptosis, autophagy, A549 cells, $\mathrm{PI} 3 \mathrm{~K} / \mathrm{Akt} / \mathrm{mTOR}$ rapamycin (mTOR) pathway was observed. It is worth noting that the inhibition of mTOR by rapamycin or of PI3K/Akt by LY294002 augmented curcumin-induced apoptosis and autophagy, leading to significant inhibition of cell proliferation. From these findings, it can be speculated that curcumin potently inhibit the cell growth of NSCLC A549 cells through inducing both apoptosis and autophagy by inhibition of the $\mathrm{PI} 3 \mathrm{~K} / \mathrm{Akt} / \mathrm{mTOR}$ pathway. These results support the potential use of curcumin as a novel candidate in treatment of human lung cancer.

\section{Introduction}

Non-small cell lung cancer (NSCLC), capturing almost $85 \%$ of lung cancers, is one of the leading causes of cancer-related mortality all over the world (1). Although chemotherapy is currently considered a valuable treatment strategy for cancer therapy, the efficacy of it on patients with advanced lung cancer is extremely limited because of drug resistance and toxicity (2). This frustrating fact renders lung cancer research and non-toxic therapeutic drugs and new intervention targets as urgent to explore and to provide more clinical benefits for lung cancer therapy.

Programmed cell death (PCD), known to be a crucial process that has an influential role in development, differentiation, cellular homeostasis, elimination of undesired and malignant cells, including apoptosis, autophagic death and necrosis, is an important target in cancer chemotherapy. Apoptosis, namely type I PCD, featured by cell shrinkage, chromatin condensation and fragmentation followed by the formation of apoptotic bodies containing intact cytoplasmic organelles or fragments of the nucleus, is a major cytotoxic mechanism of anticancer agents (3). Autophagy, a catabolic process for the degradation and recycling of macromolecules and organelles which can be activated during stress conditions is considered as a survival mechanism induced in adverse conditions to maintain cell integrity, or conversely, as an alternative cell death pathway (namely type II PCD) (4). Beclin1 is necessary in the formation of autophagic vesicles (AVs), and its level can reflect whether autophagy occurs. During the autophagy process, the cytoplasmic form of 
microtubule-associated protein light chain 3 (LC3-I, $16 \mathrm{kDa}$ ) is processed to its membrane associated form LC3-II $(14 \mathrm{kDa})$ and recruited to the autophagosomes, simultaneously p62 will continue to be consumed. Thus, the Beclin1, LC3-II/LC3-I, and p62 are regarded as a hallmark used to evaluate the level of autophagy. Recent studies have pointed towards a complex interplay between apoptosis and autophagy involved in the process of cell death because they can occur simultaneously, sequentially, or exclusively depend on cellular exposure environment and the levels of stress involved (4-6). Accumulated evidence suggests that the two different modes of cell death may be triggered by the common upstream signals, which affect the development and therapy of cancer, such as p53, Bcl-2 and PI3K/Akt/mTOR pathway (5-8).

The PI3K/Akt/mTOR pathway plays an important role in cell proliferation, cell metabolism, angiogenesis, cell cycle progression, apoptosis and autophagy, representing one of the major survival pathways that is dysregulated in various types of human cancer, and contributing to cancer pathogenesis and therapy resistance. In most malignancies this pathway is constitutively active leading to inhibition of PCD and promotion of cell survival (9). So, inhibition of PI3K/Akt/mTOR signaling pathway may be of immense potential in causing cell death associated with apoptosis and/or autophagy. However, the detailed mechanisms of different anticancer drug treatments, especially the natural drugs, all of which may involve different PCDs to a certain extent, are still rarely understood.

Natural substances are the most reliable resources for the therapy of cancer. Curcumin, a kind of liposoluble polyphenol pigment extracted from rhizome of curcuma, having a broad range of pharmacological effects such as anti-inflammatory, anticoagulant, hypolipidemic, antioxidant, free radical scavenging, and anti-atherosclerosis. Clinical trials have shown curcumin as a dietary constituent with demonstrated anti-carcinogenic capability, which is safe and well tolerated in humans $(10,11)$. Literature points to the fact that curcumin is capable of hindering the growth of multiple cancer lines in vitro and in vivo through effect on many different signaling pathways (12-14) indicating its potential clinic application in cancer control. A recent report highlighted that curcumin-induced cytotoxicity is attributable to apoptosis but not autophagy in human lung adenocarcinoma cells (15). In another study, it was shown that turmeric toxicity associated with autophagy degradation of anti-apoptosis in A431 epidermoid cancer cells (16). Based on our early studies, the results have been suggested that the promotion of lung cancer cell autophagy activity induced by curcumin even inducing autophagic death is a potential tumor treatment (17). However, the effect of apoptosis is a legacy question. No consensus has been reached yet for the interpretation of the effects of curcumin on the underlying mechanisms and the role of curcumin in inducing various PCDs in human lung cancer cells remains to be defined. Therefore, studies on the two modes of PCD related to curcumin as well as exploration of regulating function of $\mathrm{PI} 3 \mathrm{~K} / \mathrm{Akt} / \mathrm{mTOR}$, which is an essential factor in the determination of the overall fate of tumor cells, will help us to further understand their roles in tumorigenesis and provide new ideas for the treatment of lung cancer.
In the present study, the antitumor activity of curcumin especially its underlying molecular mechanism of action with apoptosis and autophagy were investigated in NSCLC A549 cells.

\section{Materials and methods}

Materials. The NSCLC A549 cells were obtained from the First Affiliated Hospital of Xi'an Jiaotong University as a gift. The curcumin, MTT, rapamycin, and LY294002 were purchased from Sigma (San Francisco, CA, USA). Roswell Park Memorial Institute culture medium (RPMI-1640) and penicillin-streptomycin were purchased from Hyclone Co. (Logan, USA). Fetal bovine serum (FBS; Biological Industries, Kibbutz Beit-Haemek, Israel). Dimethyl sulphoxide (DMSO) was purchased from Amresco (Houston, TX, USA). The Annexin V-fluorescein isothiocyanate (FITC)/propidium iodide (PI) apoptosis detection kit was purchased from 7Sea Pharmatech Co., Ltd. (Shanghai, China). TRIzol reagent was purchased from Life Technologies (Carlsbad, CA, USA). The Revert Aid First Strand cDNA Synthesis kit was purchased from Thermo Fisher Scientific (Waltham, MA, USA). FastStart Universal SYBR Green Master (Rox) was purchased from Roche (Basel, Switzerland). The Pierce bicinchoninic acid (BCA) protein assay kit was purchased from Merck (Darmstadt, Germany). The skim milk was purchased from Wandashan Dairy Co., Ltd. (Heilongjiang, China). Goat anti-rabbit IgG-HRP, Akt (60 kDa), p-Akt (60 kDa), mTOR (289 kDa), p-mTOR (289 kDa), GAPDH (38 kDa) antibodies were purchased from Abcam (Cambridge, MA, USA). The antibodies against Beclin1 (60 kDa), LC3 (LC3-II, $14 \mathrm{kDa}$ and LC3-I, $16 \mathrm{kDa})$ and p62 (62 kDa) were purchased from Cell Signaling Technology Inc. (Beverly, MA, USA).

Curcumin, rapamycin, and LY294002 were dissolved into micro-DMSO stock solution and then RPMI-1640 culture medium was added to the desired concentration, waiting to be used. DMSO is a control for the entire study at a final concentration of $<0.1 \%$.

\section{Methods}

Cell culture. A549 cells were cultured in RPMI-1640 medium supplemented with $10 \%$ (v/v) FBS, $1 \%$ penicillin-streptomycin and specifically maintained in a $5 \% \mathrm{CO}_{2}, 95 \%$ air humidified incubator at $37^{\circ} \mathrm{C}$. Taking the logarithmic growth phase of the A549 cells for following tests and the cells were seeded and adhered on petri dishes for $24 \mathrm{~h}$ before starting the treatment. A549 cells were exposed to curcumin at the indicated concentrations and time periods in each experiment. The rapamycin and LY294002 were widely used as blockers of mTOR and PI3K/Akt, respectively. When co-cultured with curcumin the cells were pre-treated with rapamycin of $40 \mu \mathrm{M}$ or LY294002 of $20 \mu \mathrm{M}$ for $3 \mathrm{~h}$ based on a large number of references $(6,18)$ and our pre-experimental results.

Cell viability assay. The cytotoxic activity of different stimulus in A549 cells was measured by 3-(4,5-dimethylthiazol-2-yl)-2,5-diphenyltetrazolium bromide (MTT) assay. A549 cells were seeded in 96-well plates at a density of $5 \times 10^{4}$ cells/well for $24 \mathrm{~h}$ before challenged by different concentrations of curcumin for $24,48,72$ and $96 \mathrm{~h}$. Or the cells 
were pretreated with $40 \mu \mathrm{M}$ rapamycin or $20 \mu \mathrm{M}$ LY294002 for $3 \mathrm{~h}$ and subsequently with or without $40 \mu \mathrm{M}$ curcumin for $48 \mathrm{~h}$. Each 96-well plate was set up with control (cells-only) and zero-adjustment (medium only). Additionally, then washed once and incubated with $20 \mu \mathrm{l} \mathrm{MTT}(5 \mathrm{mg} / \mathrm{ml})$ at $37^{\circ} \mathrm{C}$ for $4 \mathrm{~h}$. Carefully aspirating the liquid, the purple formazan crystals were dissolved in $150 \mu \mathrm{l}$ DMSO. Shaking $5 \mathrm{~min}$, the absorbance of each well was read with the microplate reader (Infinire M200; Tecan Group Ltd., Mannedorf, Switzerland) at $570 \mathrm{~nm}$. Assays were performed in triplicate on three independent experiments. Cell viability rate $(\%)=($ experimental group OD-zero adjustment group OD)/(control group OD-zero adjustment group OD) x100\%.

Apoptosis assay. The Annexin V-FITC/PI detection kit was used for the determination of cell apoptosis. A549 cells $\left(4 \times 10^{5}\right.$ cells/well) were seeded in 6 -well tissue culture plates followed by exposure with curcumin $(0-40 \mu \mathrm{M})$ as well as co-incubation with curcumin $(40 \mu \mathrm{M})$ and rapamycin $(40 \mu \mathrm{M})$ or curcumin $(40 \mu \mathrm{M})$ and LY294002 $(20 \mu \mathrm{M})$ for $48 \mathrm{~h}$ at $37^{\circ} \mathrm{C}$ and then were collected, washed once with cold phosphate-buffered saline (PBS), then re-suspended in $400 \mu \mathrm{l}$ binding buffer at a concentration of $1 \times 10^{6}$ cells $/ \mathrm{ml}$. After $5 \mu \mathrm{l}$ of Annexin V-FITC was added, the cells were incubated for $15 \mathrm{~min}$ at room temperature in the dark. Then $10 \mu \mathrm{l}$ of PI (propidium iodide) was added and the cells were incubated for $5 \mathrm{~min}$ at $4^{\circ} \mathrm{C}$ in the dark. The rate of apoptosis was immediately analyzed by a flow cytometer (Guava ${ }^{\circledR}$ easy Cyte HT; Merck Millipore, Billerica, MA, USA). Annexin V-FITC-positive cells were considered to be undergoing apoptosis and those negative for FITC were considered to be alive.

Monodansylcadaverine (MDC) labeling. Formating and promoting the AVs is one of the characters of autophagy (19). To detect autophagy, MDC labeling was performed, which could infer the activation of autophagy from the changes in fluorescent particles. Collecting the logarithmic growth phase A549 cells seeded at $3 \times 10^{4}$ cells per well in 24-well culture plates and treated with $0 \mu \mathrm{M}$ curcumin (control), $40 \mu \mathrm{M}$ curcumin, rapamycin+curcumin $(40 \mu \mathrm{M}), \mathrm{LY} 294002+$ curcumin $(40 \mu \mathrm{M})$ respectively. MDC (50 $\mu \mathrm{M})$ was added to living cells $48 \mathrm{~h}$ after different treatments. The cells were then incubated for $15 \mathrm{~min}$ at $37^{\circ} \mathrm{C}$ and $5 \% \mathrm{CO}_{2}$ in the dark, washed twice with PBS, and the anti-quencher was added. Visualizing and imaging quickly with UV excitation by an inverted fluorescence microscope (Nikon Eclipse Ti; Nikon, Tokyo, Japan) . In order to quantify MDC staining, the relative MDC fluorescence intensity of the different treatment groups were measured by the Image-Pro Plus. The experiment was repeated three times.

Quantitative real-time PCR. The A549 cells ( $2 \times 10^{6}$ cells/well) were seeded in 6-well tissue culture plates followed by exposure to curcumin $(0-40 \mu \mathrm{M})$ or co-incubation with curcumin $(40 \mu \mathrm{M})$ and rapamycin $(40 \mu \mathrm{M})$ or curcumin $(40 \mu \mathrm{M})$ and LY294002 $(20 \mu \mathrm{M})$ for $48 \mathrm{~h}$. Then total RNA was isolated with Trizol reagent from A549 cells and $2 \mu \mathrm{g}$ of the total RNA was reverse-transcribed into cDNA with the RevertAid First Strand cDNA Synthesis kit according to the manufacturer's instructions. Quantitative real-time PCR (qPCR) was performed with a FastStart Universal SYBR Green Master (Rox) kit. Each sample was run in triplicate in final volume of $25 \mu \mathrm{l}$ containing $2.5 \mu \mathrm{l}$ first-strand cDNA, $1 \mu \mathrm{l}(7.5 \mu \mathrm{M})$ of each primer (purchased from Oke Dingsheng Biological Technology Co., Ltd., Beijing, China), $12.5 \mu \mathrm{l}$ of $2 \mathrm{X}$ Fast Start Universal SYBR Green Master (ROX) and $8 \mu$ l distilled water. Cycling parameters of the qPCR were as follows: 1 cycle at $95^{\circ} \mathrm{C}$ for $10 \mathrm{~min}$, followed by 40 cycles at $95^{\circ} \mathrm{C}$ for $15 \mathrm{sec}, 60^{\circ} \mathrm{C}$ for $60 \mathrm{sec}$ in the Step One Plus fluorescence quantitative PCR instrument (Applied Biosystems; Thermo Fisher Scientific, Inc., Waltham, MA, USA). After the reaction, the results were obtained by $\triangle \triangle \mathrm{CT}$ method. Primer sequences were: Akt forward, 5'-CAAGTCCTTGCTTTCAGGGC-3' and reverse, 5'-ATACCTGGTGTCAGTCTCCGA-3' (184-bp product); mTOR forward, 5'-AACCTCCTCCCCTCCAATGA-3' and reverse, 5'-CTCACGGAGAACCAGGACAG-3' (186-bp product); GAPDH forward, 5'-CAAGGTCATCCATGACAA CTTTG-3' and reverse, 5'-GTCCACCACCCTGTTGCTGTA G-3' (496-bp product). Amplification of housekeeping gene GAPDH was taken as an endogenous control.

Western blot analysis. A549 cells were seeded in culture dish at a density of $2 \times 10^{6}$ cells/well and then incubated with or without curcumin $(0-40 \mu \mathrm{M}, 48 \mathrm{~h})$ in the presence or absence of various inhibitors ( $40 \mu \mathrm{M}$ rapamycin or $20 \mu \mathrm{M}$ LY294002). The cells were lysed in the radio-immunoprecipitation assay buffer (RIPA). Proteins were quantified using a BCA protein assay kit. Equal amount $(20 \mu \mathrm{g})$ of protein samples were subjected to $12 \%$ sodium dodecyl sulfate-polyacrylamide gel electrophoresis (SDS-PAGE) and further transferred to polyvinylidene fluoride (PVDF) membranes which were soaked with methanol for $30 \mathrm{sec}$. After blocking with 5\% bovine serum albumin for $1 \mathrm{~h}$ at room temperature and probed with the indicated antibodies. The membrane was washed thrice with TBST and incubated with goat anti-rabbit IgG conjugated to HRP. The membranes were visualized using the electrochemiluminescence and quantitated using the Image-Pro Plus6.0 software. Equal loading was assessed by GAPDH as internal control for western blotting.

Statistical analysis. SPSS18.0 statistical software was used for data analysis. The data are presented as mean \pm SEM. The one-way ANOVA were used to analyze data. Comparisons between groups were done using the Dunnett's t-test $\left({ }^{*} \mathrm{P}<0.05\right.$, ${ }^{* *} \mathrm{P}<0.01$ or $\left.{ }^{\#} \mathrm{P}<0.05,{ }^{\# \#} \mathrm{P}<0.01\right)$.

\section{Results}

Curcumin exhibits antiproliferative activity against human lung cancer A549 cells. In the first place, the toxicity of curcumin treatment at concentration ranging from $0-40 \mu \mathrm{M}$ for different time period $(24,48,72$ and $96 \mathrm{~h}$ ) was assayed by using an MTT test on human lung cancer A549 cells. As is shown in Fig. 1 the growth inhibition of curcumin on the cells was manifested in a dose- and time-dependent manner. Of note, A549 cell viability could be obviously reduced with an incubation period for $48 \mathrm{~h}$ at 20 and $40 \mu \mathrm{M}$ of curcumin consistent with our previous findings (17) selected as an effective condition for the subsequent studies to elucidate the underling molecular mechanisms of curcumin. 


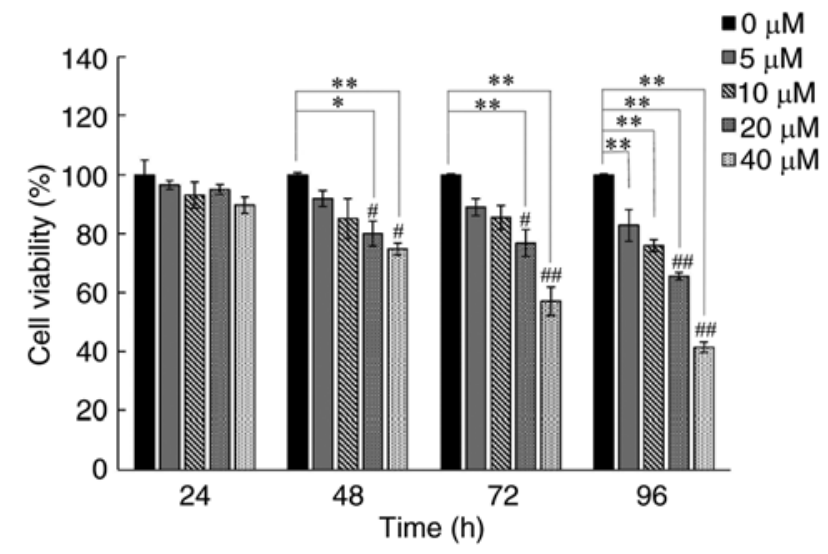

Figure 1. Curcumin inhibits cell proliferation in human lung cancer A549 cells, reflected by the results of MTT assay. It was shown that curcumin was able to inhibit the growth of A549 cells in a concentration-and time-dependent manner. Data are represented as mean \pm SEM, in three separate experiments performed in triplicate. ${ }^{*} \mathrm{P}$-values $<0.05$ and ${ }^{* *} \mathrm{P}$-values $<0.01$ for $(0 \mu \mathrm{M})$ vs. $(5,10,20$ or $40 \mu \mathrm{M}),{ }^{\#} \mathrm{P}$-values $<0.05$ and ${ }^{\# \#} \mathrm{P}$-values $<0.01$ for $(24 \mathrm{~h})$ vs. $(48$, 72 or 96 h).

Apoptotic cell death is induced after treatment with curcumin in A549 cells. Next, Annexin V-FITC and PI staining were used to examine whether this inhibitory effect of curcumin on cell ability was related to the induction of apoptosis. Apoptotic or necrotic cells were detected after treated with curcumin of increasing concentrations for $48 \mathrm{~h}$. The apoptosis phenomenon was observed in cells exposed to different doses of curcumin, whereas necrotic population was almost negligible. As shown in Fig. 2, the apoptotic (Annexin V-positive) cell populations (early and late apoptosis) were increased to 20.91 and $23.68 \%$ after treatment of A549 cells with 20 and $40 \mu \mathrm{M}$ curcumin, respectively, with a 1.35 - and 1.53 -fold increase, respectively, compared with control cells. These data suggested that curcumin resulted in a dose-dependent increase in the apoptotic cell death in human lung cancer A549 cells when used for
$48 \mathrm{~h}$. Taken together, these results revealed its central role in regulating apoptosis mediated by curcumin.

Curcumin induces autophagy in A549 cells. Autophagy, one of the most studied fields in cancer biology, has been verified by using a variety of methods. MDC is a luminescent dye that can be absorbed by cells and displayed on AVs. The activation of autophagy was inferred from changes in fluorescent particles infected with MDC first. After curcumin treatment MDC staining showed that the relative fluorescence intensity and density of cells was significantly increased compared with control cells, indicating the occurrence of autophagy (Fig. 3A and B). This was the result expected and consistent with our previous study that curcumin enhanced autophagy in a dose-dependent manner in A549 cells (17).

Taking into account the non-specificity of MDC staining, the protein levels of autophagy markers Beclin1 $(60 \mathrm{kDa})$, LC3-II (14 kDa), LC3-I (16 kDa), and p62 (62 kDa) were detected by western blotting. As shown in Fig. 3C, curcumin administration dramatically increased Beclin1 and LC3-II expression, decreasing p62 protein level. As expected, the ratio of LC3-II/LC3-I was enhanced (Fig. 3C and D) in a dose-dependent manner. All the results above provided real evidence for curcumin-induced autophagy of A549 cells.

\section{Curcumin blocks the PI3K/Akt/mTOR signal transduction} pathway. Thus far our results revealed that curcumin could substantially induce both apoptosis and autophagy. Given the critical role of PI3K/Akt/mTOR pathway in controlling cell survival/death in cancer cells, including lung cancer cells (20-22), we investigated whether curcumin induced apoptosis and autophagy via the inhibition of PI3K/Akt/mTOR signaling pathway.

To determine the role of this way, two critical molecules, Akt and mTOR, were examined. The expression levels of Akt and mTOR mRNA was detected in A549 cells by qRT-PCR (Fig. 4A and B). As expected, compared with the control group

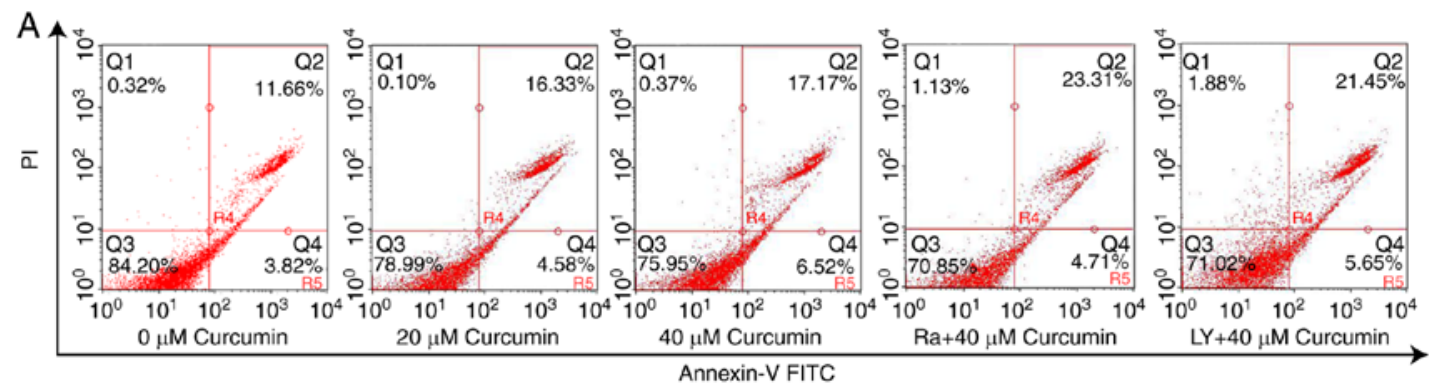

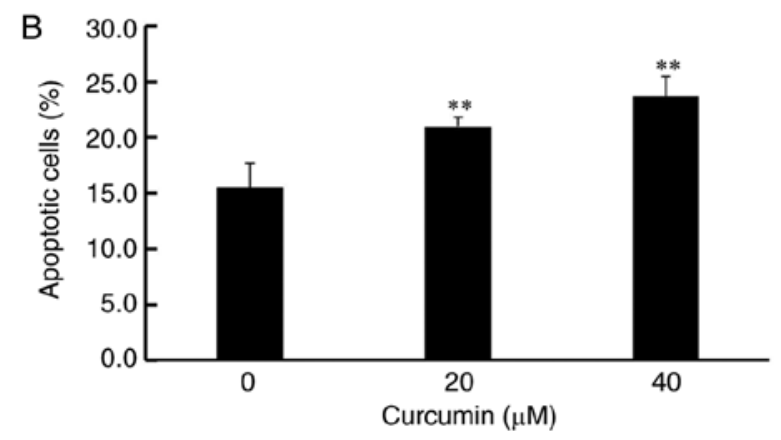

Figure 2. Apoptotic cell death in A549 cells determined by Annexin V-FITC/PI double staining and flow cytometry. A549 cells were treated with curcumin at the indicated concentrations for $48 \mathrm{~h}$. When co-cultured with curcumin the cells were pre-treated with rapamycin (Ra) of $40 \mu \mathrm{M}$ or LY294002 (LY) of $20 \mu \mathrm{M}$ for $3 \mathrm{~h}$. (A) Flow cytometry plots show specific cell populations (Q1-necrosis, Q2-late apoptosis, Q3-live, Q4-early apoptosis) in A549 cells. (B) Bar graph indicates the percentage of apoptotic cell populations (early and late apoptosis) showing a dose-dependent increase in the apoptotic cell death with curcumin treatment for $48 \mathrm{~h}$. Data are represented as mean $\pm \mathrm{SEM}$, in three separate experiments performed in triplicate. ${ }^{* *} \mathrm{P}$-values $<0.01$. 

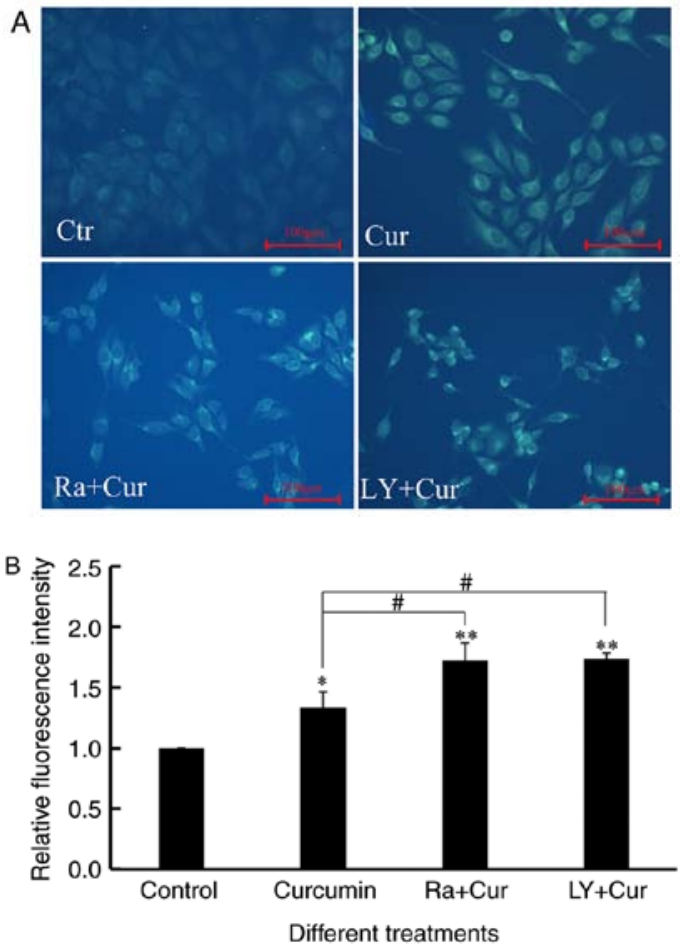

C
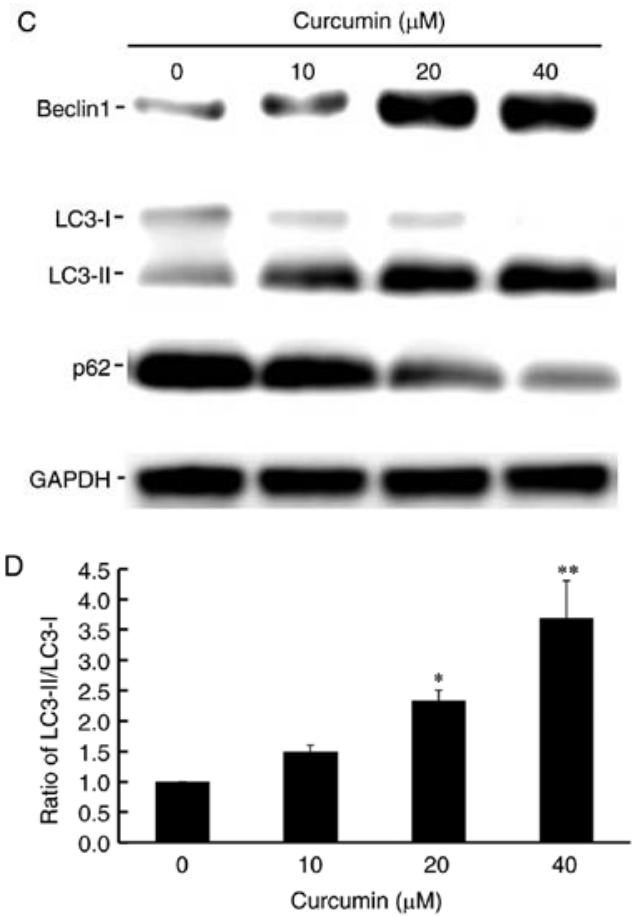

Figure 3. Autophagic induction is detected in A549 cells. (A) Microscopic detection of autophagic vesicles (AVs) in A549 cells by MDC staining. Contro (Ctr) is concentration $0 \mu \mathrm{M}$ of curcumin. The image showed that curcumin (Cur) at $40 \mu \mathrm{M}$ for $48 \mathrm{~h}$ administration increased the fluorescence intensity and density which is evidence of autophagy induction by curcumin and either rapamycin (Ra) or LY294002 (LY) pre-treatment potentiated autophagy in A549 cells in comparison with curcumin treatment alone. Images were taken with a fluorescence microscope (magnification, x200; scale bar, $100 \mu \mathrm{m}$ ). (B) The relative MDC fluorescence intensity of the different treatment groups have been measured by Image-Pro Plus suggesting the induction of autophagy. (C) Proteins of Beclin1, LC3-II, LC3-I, and p62 were detected by western blot assay. GAPDH was used as loading control. (D) Bar graphs indicate the ratio of LC3-II/LC3-I which was enhanced in a dose-dependent manner indicating autophagy induction. Data are represented as mean \pm SEM, in three separate experiments performed in triplicate. Comparison with control group, ${ }^{*} \mathrm{P}$-values $<0.05$ and ${ }^{* *} \mathrm{P}$-values $<0.01$. Comparison with $40 \mu \mathrm{M}$ curcumin treatment group, ${ }^{\text {"P}} \mathrm{P}$-value $<0.05$.
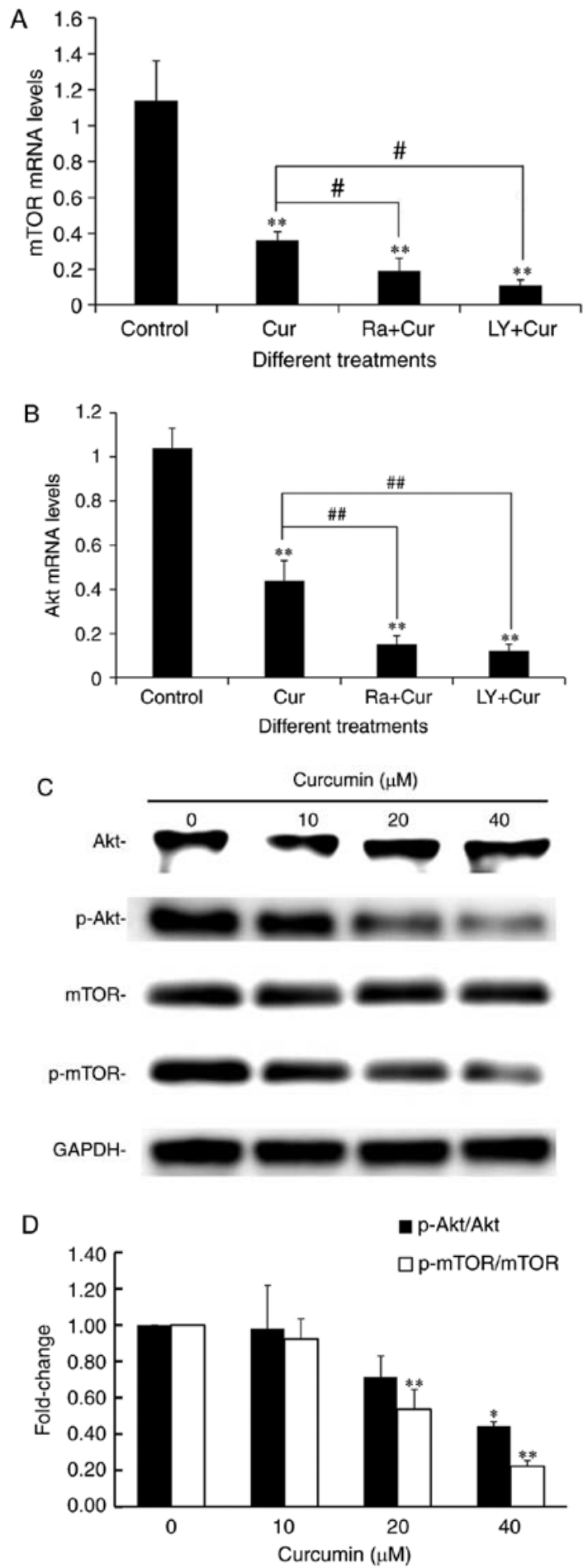

Figure 4. Signal transduction pathway of PI3K/Akt/mTOR was downregulated by curcumin treatment. A549 cells were treated with curcumin (Cur) at the indicated concentrations $(0-40 \mu \mathrm{M})$ for $48 \mathrm{~h}$. When co-cultured with curcumin (Cur) the cells were pre-treated with rapamycin (Ra) of $40 \mu \mathrm{M}$ or LY294002 (LY) of $20 \mu \mathrm{M}$ for $3 \mathrm{~h}$. Control is concentration $0 \mu \mathrm{M}$ of curcumin. (A and B) The expression of two key proteins mTOR and Akt mRNA expression associated with $\mathrm{PI} 3 \mathrm{~K} / \mathrm{Akt} / \mathrm{mTOR}$ signaling pathways, detected by the quantitative real-time PCR test. The qRT-PCR results showed that mTOR or Akt mRNA expression in cur, LY2+Cur and ra+Cur groups were significantly decreased as compared with control group. (C and D) Proteins of total and phosphorylation mTOR as well as Akt were detected by western blot assay. GAPDH was used as loading control. Bar graphs indicate the ratio of $\mathrm{p}-\mathrm{Akt} / \mathrm{mTOR}$ and $\mathrm{p}-\mathrm{mTOR} / \mathrm{mTOR}$, respectively. A dose-dependent inhition of Akt phosphorylation and mTOR phosphorylation were observed. Data were represented as mean \pm SEM, in three separate experiments performed in triplicate. Comparison with control group, "P-values $<0.05$ and ${ }^{* *} \mathrm{P}$-values $<0.01$. Comparison with $40 \mu \mathrm{M}$ curcumin treatment group, ${ }^{\text {PP-value }}<0.05$ and ${ }^{\# \#} \mathrm{P}$-value $<0.01$. 


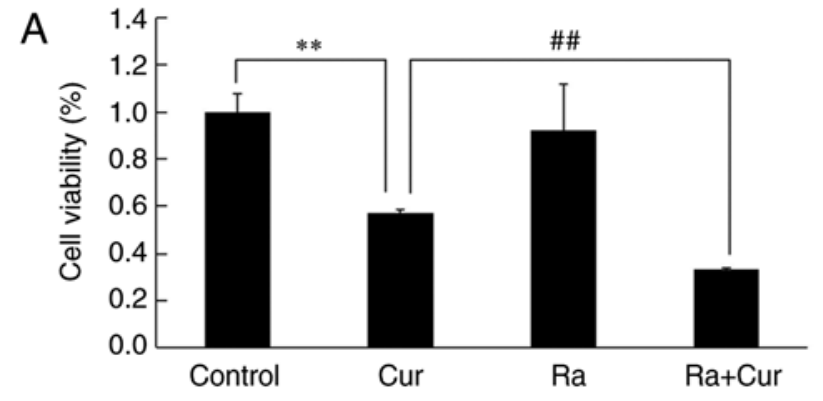

Different treatments
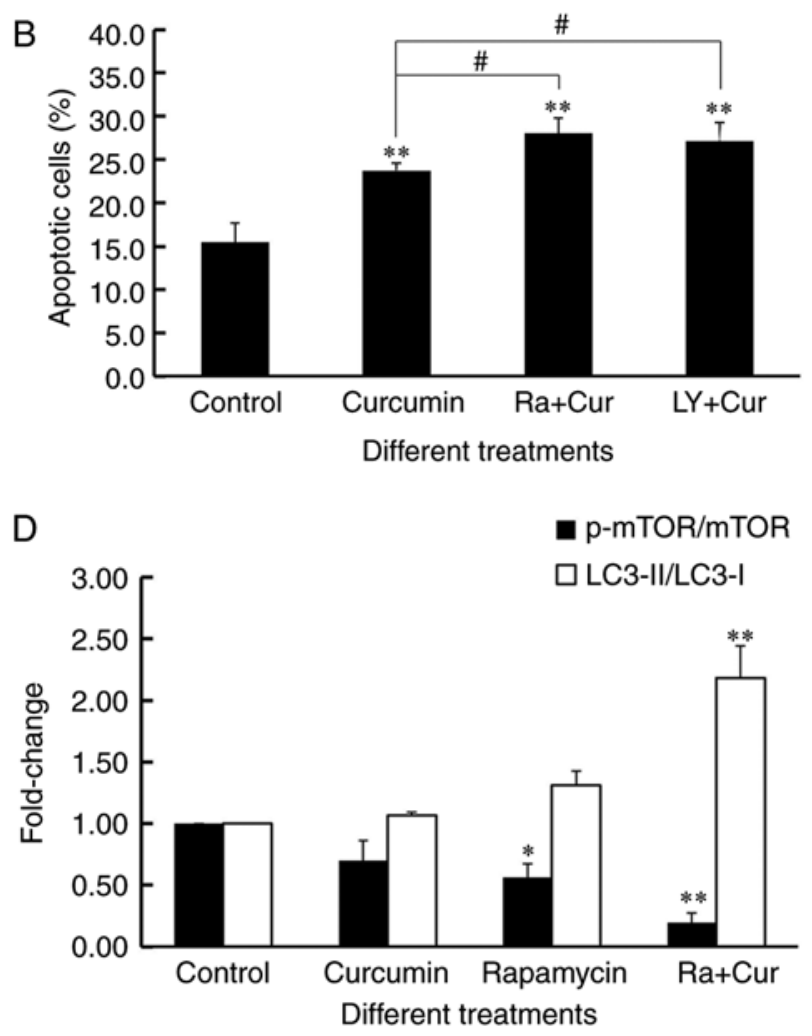

F

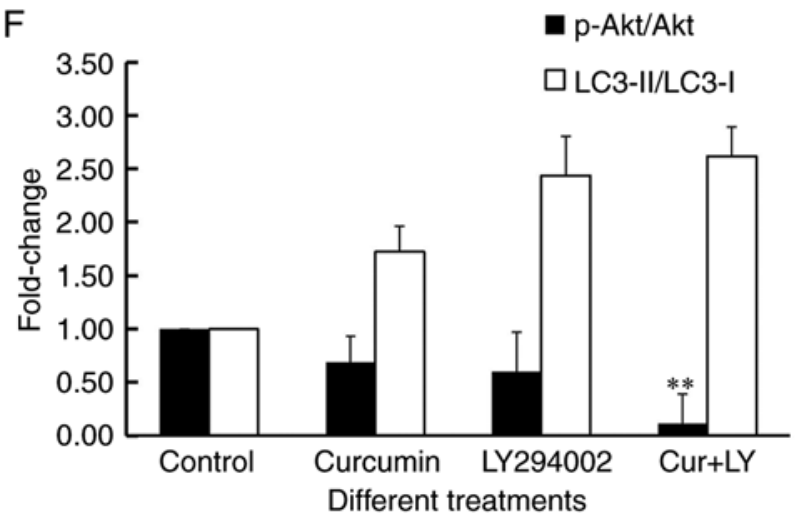

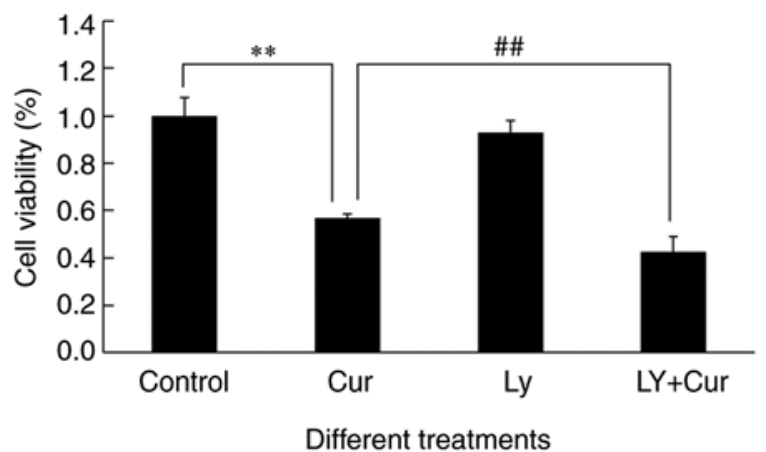

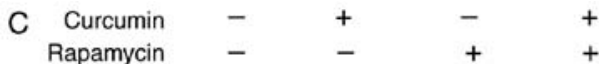
mTORp-mTOR-
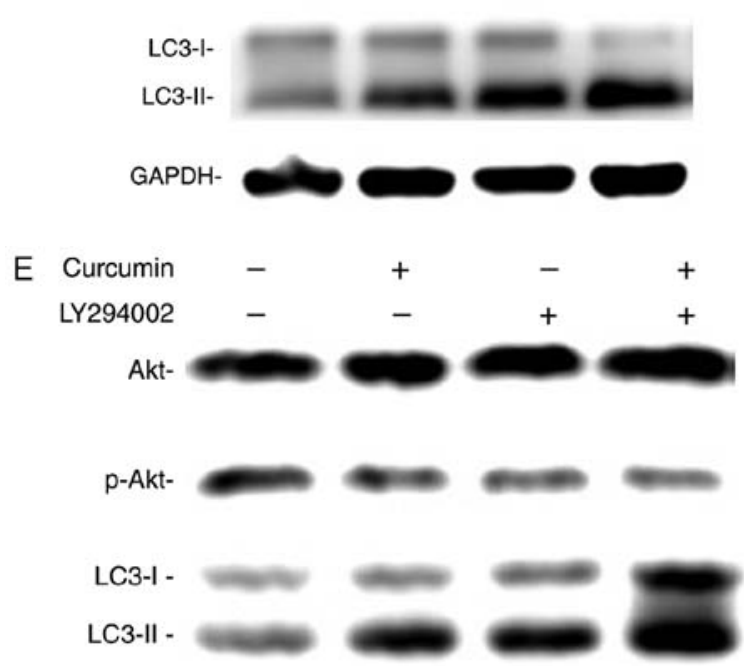

GAPDH-

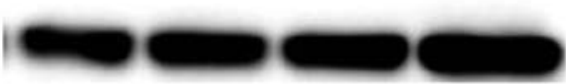


(1.14 \pm 0.22$), 40 \mu \mathrm{M}$ of curcumin treated A549 cells $48 \mathrm{~h}$, greatly reduced the transcription level of mTOR $(0.36 \pm 0.05)(\mathrm{P}<0.01)$, as indicated in Fig. 4A. As shown in Fig. 4B, the expression of Akt mRNA in curcumin group $(0.44 \pm 0.09)$ was significantly lower than that in control $(1.04 \pm 0.09)(\mathrm{P}<0.01)$. In order to further ascertain and identify the role of mTOR and Akt in the curcumin-modulation PI3K/Akt/mTOR signaling in A549 cells, the effect of rapamycin and LY294002 were detected. The rapamycin and LY294002 were widely used as inhibitors of mTOR and PI3K/Akt, respectively $(23,24)$. Similar to the effect of curcumin, there was a more significant inhibitory effect on mTOR and Akt mRNA levels when it combined with rapamycin or LY294002 compared with curcumin treatment alone (Fig. 4A and B).

Eukaryotic gene expression and regulation is complex, mRNA levels can not fully represent the expression of protein. Thus, the protein expression of important signaling molecules such as p-Akt, Akt, p-mTOR, and mTOR was determined by western blotting. As shown in Fig. 4C, dose-dependent inhition of Akt phosphorylation and mTOR phosphorylation were observed when A549 cells were treated with curcumin for $48 \mathrm{~h}$. To be more intuitive, the inhibition of PI3K/Akt/mTOR pathway was judged by the ratio of p-Akt to total Akt as well as p-mTOR to total mTOR, which was consistent with the above results (Fig. 4D). These data together imply that the cytotoxicity of curcumin may be related to the PI3K/Akt/mTOR pathway inhibition.

Pre-treatment with rapamycin or LY294002 enhances curcumin-induced apoptosis and autophagy with PI3K/Akt/mTOR pathway suppression, reducing cell viability in A549 cells. Above observations prompted to infer that downregulation of PI3K/Akt/mTOR might have a crucial role in curcumin-induced cytotoxicity due to apoptosis and autophagy. Therefore, the cell viability was examined by MTT. To determine that the curcumin-induced apoptosis and autophagy occurred via the PI3K/Akt/mTOR signaling pathway, we employed mTOR blocker rapamycin and PI3K/Akt inhibitor LY294002. The MTT assay showed that inactivation of PI3K/Akt or inactivation of mTOR tremendously sensitized A549 cells toward cytotoxicity of curcumin (Fig. 5A). The level of curcumin-induced cell death was enhanced when the PI3K/Akt/mTOR signal transduction pathway was blocked, which may be related to the induction of PCDs.

As shown in Figs. 2A and 5B, the Annexin V-FITC/PI double stained assays revealed that the cell pre-treatment with rapamycin of $40 \mu \mathrm{M}$ or LY294002 of $20 \mu \mathrm{M}$ resulted in a significantly greater number of apoptotic (Annexin V-positive) cells than treatment with curcumin alone. MDC labeling analyses were done to detect the acidic vacuoles. Similarly, fluorescence microscopy shows significant increase in the number of MDC-labeled vacuoles (Fig. 3A and B) in cells exposed to rapamycin or LY294002 co-cultured with curcumin compared with curcumin treatment alone. Compared to curcumin alone, the ratio of LC3-II/LC3-I was significantly enhanced (Fig. 5C and D) in a dose-dependent manner when cells were pretreated with rapamycin, resulting in consistent results when curcumin was co-cultured with LY294002 (Fig. 5E and F), suggesting autophagy was clearly induced. The above results illustrated that combined treatment of PI3K/Akt or mTOR blockers significantly enhanced apoptosis and autophagy, accompanied by the inhibition of $\mathrm{PI} 3 \mathrm{~K} / \mathrm{Akt} / \mathrm{mTOR}$ pathway can be derived from the ratio of p-mTOR to total mTOR (Fig. 5C and D) as well as p-Akt to total Akt (Fig. 5E and F) downgrade. These data might support the idea that cytotoxicity derived from apoptosis and autophagy in A549 cells by curcumin proceeds through $\mathrm{PI} 3 \mathrm{~K} / \mathrm{Akt} / \mathrm{mTOR}$ inhibition. Thus, our findings unequivocally substantiated the fact that PI3K/Akt/mTOR is involved in the regulation of both apoptosis and autophagy induced by curcumin. Inhibition of mTOR promoted the development of both autophagy and apoptosis.

The present study collectively showed that curcumin has a similar function as the PI3K/Akt/mTOR pathway inhibitor resulting in varying degrees of reduction of the Akt phosphorylation and mTOR phosphorylation as well as mRNA expression. From the present results, our data indeed identified that curcumin exerts cytotoxic effect on A549 cells by inhibiting the PI3K/Akt/mTOR pathway to promote apoptosis and autophagy induced, indicating that PI3K/Akt/mTOR signal transduction pathway is a key pathway involved in the role of curcumin in lung cancer, and might be also the main target of curcumin.

\section{Discussion}

It is known that tumor is a disease in which cell proliferation and death are imbalanced and the lung cancer is the most prevalent malignant tumor. Recently, many natural plant agents possessing availability and relatively low toxicity have been reported to treat cancer by inhibiting cell proliferation (25). One of the liposoluble polyphenol pigments, curcumin, has previously been reported to have effects on suppressing the growth of a variety of cancer lines (12-14). This sequence of events is strongly supported by the results of the present study which disseminated evidence that induction of multiple modes of cell death and potential mechanisms in the context of cytotoxicity induced by curcumin in human lung cancer A549 cells.

The anti-proliferation of curcumin in human lung adenocarcinoma A549 cell line was confirmed, showing that curcumin is a potent inhibitor of lung cancer cells in vitro. Also, evidence was obtained that treatment with curcumin at different concentration and time range selectively decreased the proliferation of the cells in a dose- and time-dependent manner (Fig. 1). Nevertheless, we endeavoured to further explore the molecular process underlying curcumin induced cell death.

Various forms of PCD are increasingly involved in anticancer treatment, and the complex interplay among them is critical to the ultimate fate of proliferating cells. Whether curcumin possesses the ability to induce multiple PCDs including apoptosis and autophagy in human lung cancer A549 cells, has aroused our interest. Induction of apoptosis, namely type I PCD, was a highly desirable characteristic for screening of chemotherapeutic drugs (6). Our results showed that curcumin proved to be a potent inducer of apoptosis in A549 cells as evidenced by an dose-dependent increase in Annexin V-positive cell populations (Fig. 2). Next, the events 
governing the autophagy process was studied. To elucidate the autophagy (namely type II PCD) induction by curcumin in A549 cells, MDC labeling analysis was done to detect the AVs suggesting an enhanced increase of the fluorescent structures compared with control. Evidence has been captured that curcumin administration dramatically increased Beclin1 and LC3-II expression, decreased p62 protein level, indicating that autophagy was induced (Fig. 3).

In recent years, an increasing number of research shows that autophagy may play a dual role in tumors, likely this could be a survival or cell death mechanism. Focusing on our earlier study on the role of curcumin-induced autophagy in lung cancer A549 cells, it was demonstrated that autophagy was the antitumor mechanism of curcumin rather than a protective mechanism of the A549 cells itself when treated with curcumin (17). Based on the above results, it was determined that curcumin-exposure within a limited range could induced apoptosis and autophagic death in A549 cells. These data clearly expressed the contribution of curcumin-induced apoptosis and autophagy in the inhibition of A549 cell growth. Recent studies have pointed towards a complex interplay between apoptosis and autophagy involved in the process of cell death because they may act independently of one another or may function as partners in a synchronized manner $(5,6,26)$. In the debate on the intimate relationship between apoptosis and autophagy in different tumor cells, we considered the possibility that curcumin could inhibit the growth of human lung cancer A549 cells by concomitantly inducing apoptosis and autophagy, supporting the view of some scholars (27). However, the mechanism involved in curcumin-induced apoptosis and autophagy of A549 cells should be further explored and elucidated urgently.

Accumulated evidence suggests that apoptosis and autophagy share some common signaling pathways, such as p53, Bcl-2 and PI3K/Akt/mTOR pathway $(5-8,28,29)$. In view of the important role played by PI3K/Akt/mTOR signaling in cell growth, studies have shown that downregulation of this pathway caused cell death associated with apoptosis and/or autophagy $(30,31)$. Two key molecules, mTOR and Akt, were detected in order to determine the role of this pathway in the curcumin-treated human lung adenocarcinoma A549 cells. The present results demonstrated that the Akt phosphorylation and mTOR phosphorylation as well as the mRNA expression were significantly decreased after curcumin treatment (Fig. 4), thus indicating that curcumin blocked the PI3K/Akt/mTOR signal transduction pathways in human lung cancer A549 cells. In addition, the PI3K/Akt-specific inhibitor LY294002 and mTOR blocker rapamycin were used in order to further determine curcumin-induced apoptosis and autophagy by PI3K/Akt/mTOR signaling pathway. Some previous studies have showed that mTOR regulates apoptosis by phosphorylation of Bax to disrupt the Bad binding to Bcl-XL, and/or Blc-2 in different cancer cells (32). It should also be pointed out that mTOR, emerging as a key negative regulator of autophagy, promotes autophagy induction by dephosphorylation of Atg1, Atg13 to promote autophagosome formation (33). Our results are consistent with the above studies that p-mTOR downregulation attributed to the consequent inhibition of the PI3K/Akt/mTOR pathway, and might act as an important factor in cross talk between curcumin-induced apoptosis and autophagy.
In our study, more significant inhibition of PI3K/Akt/mTOR pathway occurred in the co-culture of curcumin and LY294002 or curcumin and rapamycin (Fig. 5). It is worth mentioning that either LY294002 or rapamycin pre-treatment not only enhanced the apoptosis-inducing activity (Fig. 5B) and anticancer efficacy of curcumin (Fig. 5A) but also potentiated autophagy induction in A549 cells in comparison with curcumin treatment alone (Fig. 5C-F). All these data further support the idea that apoptosis and autophagy activation by curcumin proceeds through PI3K/Akt/mTOR inhibition. Briefly, the induction of apoptosis and autophagy causing cell death after curcumin exposure is closely linked to the inhibition of PI3K/Akt/mTOR demonstrating this pathway plays a pivotal role in curcumin treatment of A549 cells. However, there is no doubt that the mechanism of inducing PCD is very complex, and the fate of tumor cells is not determined by only one pathway. Therefore, it is the direction of our future research to explore and elucidate the complex relationship between curcumin-induced apoptosis and autophagy as well as more detailed mechanisms in lung cancer cells.

In conclusion, the A549 cell growth inhibitory effect of curcumin was studied. Specifically, curcumin-induced apoptosis may act as autophagy partner to induce cell death, contributing to curcumin toxicity via inhibiting PI3K/Akt/mTOR pathway which has been further deciphered in human lung cancer A549 cells. The results of the study indicates curcumin may be considered as a candidate agent targeting PCD in clinical practice and inhibiting the PI3K/Akt/mTOR signaling pathway which has been suggested as a potential therapeutic target in NSCLC.

\section{Acknowledgements}

This study was supported by the National Natural Science Foundation of China (grant no. 81172598).

\section{References}

1. Ramalingam SS, Owonikoko TK and Khuri FR: Lung cancer: New biological insights and recent therapeutic advances. CA Cancer J Clin 61: 91-112, 2011.

2. Reck M,Heigener DF, Mok T, Soria JC and Rabe KF: Management of non-small-cell lung cancer: Recent developments. Lancet 382: 709-719, 2013.

3. Kim WK, Pyee Y, Chung HJ, Park HJ, Hong JY, Son KH and Lee SK: Antitumor activity of spicatoside a by modulation of autophagy and apoptosis in human colorectal cancer cells. J Nat Prod 79: 1097-1104, 2016.

4. White E: Deconvoluting the context-dependent role for autophagy in cancer. Nat Rev Cancer 12: 401-410, 2012.

5. Ge J, Liu Y, Li Q, Guo X, Gu L, Ma ZG and Zhu YP: Resveratrol induces apoptosis and autophagy in T-cell acute lymphoblastic leukemia cells by inhibiting Akt/mTOR and activating p38-MAPK. Biomed Environ Sci 26: 902-911, 2013.

6. Kumar D, Das B, Sen R, Kundu P, Manna A, Sarkar A, Chowdhury C, Chatterjee M and Das P: Andrographolide analogue induces apoptosis and autophagy mediated cell death in U937 cells by inhibition of PI3K/Akt/mTOR pathway. PLoS One 10: e0139657, 2015.

7. Saiki S, Sasazawa Y, Imamichi Y, Kawajiri S, Fujimaki T, Tanida I, Kobayashi H, Sato F, Sato S, Ishikawa K, et al: Caffeine induces apoptosis by enhancement of autophagy via PI3K/Akt/mTOR/p70S6K inhibition. Autophagy 7: 176-187, 2011.

8. Zhou ZW, Li XX, He ZX, Pan ST, Yang Y, Zhang X, Chow K, Yang T, Qiu JX, Zhou Q, et al: Induction of apoptosis and autophagy via sirtuin1- and PI3K/Akt/mTOR-mediated pathways by plumbagin in human prostate cancer cells. Drug Des Devel Ther 9: 1511-1554, 2015. 
9. Gills JJ, Lopiccolo J and Dennis PA: Nelfinavir, a new anti-cancer drug with pleiotropic effects and many paths to autophagy. Autophagy 4: 107-109, 2008.

10. Gupta SC, Patchva S and Aggarwal BB: Therapeutic roles of curcumin: Lessons learned from clinical trials. AAPS J 15: 195-218, 2013.

11. Zhou GZ, Cao FK, Chang JM, Sun GC and Chen XB: Mechanism of curcumin analog MHMD-induced cell death in A549 lung cancer cells. Eur Rev Med Pharmacol Sci 18: 3134-3138, 2014.

12. Chang CC, Fu CF, Yang WT, Chen TY and Hsu YC: The cellular uptake and cytotoxic effect of curcuminoids on breast cancer cells. Taiwan J Obstet Gynecol 51: 368-374, 2012.

13. Liu D, You M, Xu Y, Li F, Zhang D, Li X and Hou Y: Inhibition of curcumin on myeloid-derived suppressor cells is requisite for controlling lung cancer. Int Immunopharmacol 39: 265-272, 2016.

14. Watson JL, Greenshields A, Hill R, Hilchie A, Lee PW, Giacomantonio CA and Hoskin DW: Curcumin-induced apoptosis in ovarian carcinoma cells is p53-independent and involves p38 mitogen-activated protein kinase activation and downregulation of $\mathrm{Bcl}-2$ and survivin expression and Akt signaling. Mol Carcinog 49: 13-24, 2010.

15. Zhang J, Zhang T, Ti X, Shi J, Wu C, Ren X and Yin $\mathrm{H}$ : Curcumin promotes apoptosis in A549/DDP multidrug-resistant human lung adenocarcinoma cells through an miRNA signaling pathway. Biochem Biophys Res Commun 399: 1-6, 2010.

16. Thongrakard V, Titone R, Follo C, Morani F, Suksamrarn A, Tencomnao $\mathrm{T}$ and Isidoro $\mathrm{C}$ : Turmeric toxicity in A431 epidermoid cancer cells associates with autophagy degradation of anti-apoptotic and anti-autophagic p53 mutant. Phytother Res 28: 1761-1769, 2014.

17. Liu F, Gao S, Yang Y, Zhao X, Fan Y, Ma W, Yang D, Yang A and Yu Y: Curcumin induced autophagy anticancer effects on human lung adenocarcinoma cell line A549. Oncol Lett 14: 2775-2782, 2017.

18. Jiao D, Wang J, Lu W, Tang X, Chen J, Mou H and Chen QY: Curcumin inhibited HGF-induced EMT and angiogenesis through regulating c-Met dependent $\mathrm{PI} 3 \mathrm{~K} / \mathrm{Akt} / \mathrm{mTOR}$ signaling pathways in lung cancer. Mol Ther Oncolytics 3: 16018, 2016.

19. Wang Y, Zhou T, Sun H and Huang B: Study on the relationship between autophagy and apoptosis in a549 cells induced by curcumin analogue EF24. Chin J Cell Biol 34: 590-596, 2012.

20. Chang L, Graham PH, Hao J, Ni J, Bucci J, Cozzi PJ, Kearsley JH and $\mathrm{Li} \mathrm{Y}$ : Acquisition of epithelial-mesenchymal transition and cancer stem cell phenotypes is associated with activation of the $\mathrm{PI} 3 \mathrm{~K} / \mathrm{Akt} / \mathrm{mTOR}$ pathway in prostate cancer radioresistance. Cell Death Dis 4: e875, 2013.

21. Graupera M and Potente M: Regulation of angiogenesis by PI3K signaling networks. Exp Cell Res 319: 1348-1355, 2013.

22. Huo R, Wang L, Liu P, Zhao Y, Zhang C, Bai B, Liu X, Shi C, Wei $\mathrm{S}$ and Zhang H: Cabazitaxel-induced autophagy via the $\mathrm{PI} 3 \mathrm{~K} / \mathrm{Akt} / \mathrm{mTOR}$ pathway contributes to A549 cell death. Mol Med Rep 14: 3013-3020, 2016.
23. Jiang LB, Cao L, Yin XF, Yasen M, Yishake M, Dong J and Li XL: Activation of autophagy via $\mathrm{Ca}(2+)$-dependent AMPK/mTOR pathway in rat notochordal cells is a cellular adaptation under hyperosmotic stress. Cell Cycle 14: 867-879, 2015.

24. Zhang DM, Liu JS, Deng LJ, Chen MF, Yiu A, Cao HH, Tian HY, Fung KP, Kurihara H, Pan JX and Ye WC: Arenobufagin, a natural bufadienolide from toad venom, induces apoptosis and autophagy in human hepatocellular carcinoma cells through inhibition of PI3K/Akt/mTOR pathway. Carcinogenesis 34: 1331-1342, 2013.

25. Gali-Muhtasib H, Hmadi R, Kareh M, Tohme R and Darwiche N: Cell death mechanisms of plant-derived anticancer drugs: Beyond apoptosis. Apoptosis 20: 1531-1562, 2015.

26. Tsai JP, Lee CH, Ying TH, Lin CL, Lin CL, Hsueh JT and Hsieh YH: Licochalcone A induces autophagy through $\mathrm{PI} 3 \mathrm{~K} / \mathrm{Akt} / \mathrm{mTOR}$ inactivation and autophagy suppression enhances Licochalcone A-induced apoptosis of human cervical cancer cells. Oncotarget 6: 28851-28866, 2015.

27. Kumar S, Guru SK, Pathania AS, Manda S, Kumar A, Bharate SB Vishwakarma RA, Malik F and Bhushan S: Fascaplysin induces caspase mediated crosstalk between apoptosis and autophagy through the inhibition of PI3K/AKT/mTOR signaling cascade in human leukemia HL-60 cells. J Cell Biochem 116: 985-997, 2015.

28. Turcotte S and Giaccia AJ: Targeting cancer cells through autophagy for anticancer therapy. Current Opin Cell Biol 22: 246-251, 2010.

29. Wang K, Liu R, Li J, Mao J, Lei Y, Wu J, Zeng J, Zhang T, Wu H, Chen L, et al: Quercetin induces protective autophagy in gastric cancer cells: Involvement of Akt-mTOR- and hypoxia-induced factor 1alpha-mediated signaling. Autophagy 7: 966-978, 2011.

30. Liu Z, Wang F, Zhou ZW, Xia HC, Wang XY, Yang YX, He ZX, Sun $T$ and Zhou SF: Alisertib induces G2/M arrest, apoptosis, and autophagy via PI3K/Akt/mTOR- and p38 MAPK-mediated pathways in human glioblastoma cells. Am J Transl Res 9: 845-873, 2017.

31. Wang F, Mao Y, You Q, Hua D and Cai D: Piperlongumine induces apoptosis and autophagy in human lung cancer cells through inhibition of PI3K/Akt/mTOR pathway. Int J Immunopathol Pharmacol 28: 362-373, 2015.

32. Tirado OM, Mateo-Lozano $\mathrm{S}$ and Notario V: Rapamycin induces apoptosis of JN-DSRCT-1 cells by increasing the Bax: $\mathrm{Bcl}-\mathrm{xL}$ ratio through concurrent mechanisms dependent and independent of its mTOR inhibitory activity. Oncogene 24: 3348-3357, 2005

33. Bilir A, Erguven M, Oktem G, Ozdemir A, Uslu A, Aktas E and Bonavida B: Potentiation of cytotoxicity by combination of imatinib and chlorimipramine in glioma. Int $\mathrm{J}$ Oncol 32: 829-839, 2008. 